\title{
Healthcare and economic burden of ANCA-associated vasculitis in Italy: an integrated analysis from clinical and administrative databases
}

\author{
Luca Quartuccio $^{1}$ [D $\cdot$ Elena Treppo ${ }^{1} \cdot$ Francesca Valent $^{2} \cdot$ Salvatore De Vita $^{1}$
}

Received: 13 May 2020 / Accepted: 2 July 2020 / Published online: 14 July 2020

(C) The Author(s) 2020

\begin{abstract}
ANCA-associated vasculitides (AAV) comprise a group of systemic vasculitides characterized by inflammation of small-sized blood vessels leading to multi-organ involvement. The worldwide annual incidence of AAV ranges from 1.2 to 3.3 cases per 100000 individuals with a prevalence of 4.6-42.1 cases per 100000 individuals. The prevalence of AAV is geographically heterogeneous; therefore, regional epidemiological studies can be more informative to improve health care systems. Even though clinicians are aware that the healthcare burden and the risk of hospitalization of AAV appear high, data on hospitalization and cost of illness due to AAV are still scarce or even lacking. This study aims to characterize the economic burden of AAV in Friuli Venezia Giulia (FVG), Italy. Thus, a retrospective study was conducted through the integration of many administrative health databases of the FVG as the source of information. From data integration, we estimated that more than two-thirds of AAV patients showed at least one hospitalization in their medical history, most frequently caused by the disease itself or superimposed infections. Around $10 \%$ of patients developed end-stage renal disease. In an 8-year follow-up, the overall healthcare cost was $€ 1,215,078$, corresponding to $€ 6,168$ patient-year. ANCA-positive patients showed much higher costs than ANCA-negative patients did. Overall, AAV are rare diseases, but imply very high healthcare costs. Early diagnosis and optimal treatment probably still remain unmet needs for AAV.
\end{abstract}

Keywords Vasculitis $\cdot$ ANCA $\cdot$ Autoantibody $\cdot$ Cost $\cdot$ Healthcare $\cdot$ Hospitalization $\cdot$ Granulomatosis $\cdot$ Polyangiitis

\section{Introduction}

ANCA-associated vasculitides (AAV) are a group of systemic vasculitides characterized by inflammation of smallsized blood vessels leading to multi-organ involvement [1-5]. AAV include three major clinic-pathologic entities: granulomatosis with polyangiitis (GPA), microscopic polyangiitis (MPA), and eosinophilic granulomatosis with polyangiitis (EGPA) [6, 7]. AAV are rare diseases, with a worldwide annual incidence ranging from 1.2 to 3.3 cases

Luca Quartuccio and Elena Treppo have equally contributed to this work.

Luca Quartuccio

luca.quartuccio@uniud.it

1 Department of Medicine (DAME), Clinic of Rheumatology, Department of Medicine (DAME), ASU FC, University of Udine, Udine, Italy

2 Igiene Ed Epidemiologia Clinica, ASU FC, Udine, Italy per 100000 individuals with a prevalence of $4.6-42.1$ cases per 100000 individuals [2]. Nevertheless, the incidence and prevalence of AAV have been progressively increasing in the last decades [2]. Among the subsets of AAV important geographic differences have been observed $[2,8]$. In Europe and Australia, a higher proportion of GPA versus MPA has been observed [2, 9]. Differently, in Asia (especially Japan), a higher proportion of MPA versus GPA has been observed [2]. The improvement in induction therapy has changed acute and life-threatening diseases to chronic ones associated with relapses, organ damage accumulation, and long-term immunosuppressive treatment side effects [2, 10-16]. Therefore, AAV shows a high healthcare burden with a high risk of hospitalization. However, data on hospitalization and the cost of illness due to AAV are still limited $[3,17]$. Herein, we report the healthcare burden and the direct costs of the illness of AAV in Friuli Venezia Giulia (FVG), a region in the northeast of Italy. Importantly, two different data sources were used and compared, i.e., administrative healthcare databases and clinical databases. Second, 
the costs of illness from a European healthcare system are information currently lacking for $\mathrm{AAV}$ in the literature.

\section{Methods}

The Regional Health Information System of FVG (about 1 200000 inhabitants) and clinical electronic chart records from our Hospital Clinic were used as the source of information for this retrospective cohort study.

The system covers the entire regional population and includes various electronic health administrative databases that can be linked with one another on an individual basis through a unique encrypted ID identifier. The database of the regional potential health care beneficiaries (including demographic information and the residential history of all of the subjects living in the region), the hospital discharge database, the pharmaceutical prescription database, and the database of exemptions from medical charges were used for this study.

The hospital discharge database includes records from all of the regional hospitals (both public and private accredited to the public health system) and those regarding admissions of regional residents to extra-regional hospitals. The pharmaceutical prescription database contains information on all of the medications prescribed by the physicians working in the public health system except those paid out-of-pocket. The database of exemptions from medical charges includes records on all of the potential health care beneficiaries who are entitled, because of low income, age, or chronic diseases, to receive free medications and outpatient specialist care. Notably, the Emergency Department (ED) database is accessible, but, contrary to the procedures for hospital admissions or outpatient ambulatory care, the ED has no standard tariff associated with healthcare episodes. Thus, we have access to ED records, but they do not include any cost information.

The Italian Ministry of Health assigns codes to all of the diseases that entitle patients to exemptions. Currently, they include approximately 100 chronic and disabling diseases including the groups of rare diseases [18], where GPA, MPA, and EGPA are identified with the following codes: RG0070, RG0020, and RG0050, respectively. In the Italian healthcare system, only the specialists have the authorization to provide the exemption codes for rare diseases, and since the year 2010, a great effort has been made in our Region to keep the rare disease registry up-to-date, with the aim of filling such gap.

The cohort included all of the subjects living in FVG who received an exemption from medical charges because of a diagnosis of either GPA, MPA, or EGPA, according to the corresponding exemption code from 2010 to 2018. The subjects were observed from the date of first release of the exemption and followed until they moved outside the region, died, the outcome of interest occurred, or December 31, 2018, whichever came first. The outcome of interest was the event of hospitalization or death. Of note, we restricted the time frame of observation to the years from 2013 to 2018 as concerns the analysis on the whole regional population, i.e., starting from the year in which the laboratory analyses were centralized in the Laboratory of the Hospital of Udine, in particular for the autoimmunity tests, while we analyzed a longer time frame (2010-2018) when considering the province of Udine (about 530000 inhabitants, the half of the whole regional population). For the best comparison, regarding the local retrospective cohort from our Clinic, we included in the analysis all the patients examined in Rheumatology Clinic of Udine (Referral Centre for AAV in FVG) who received diagnosis of GPA, MPA, and EGPA from 2010 to 2018.

\section{Statistical analysis}

The frequency distribution of the baseline cohort characteristics and events of interest was calculated. The statistical significance of differences in the variable distribution between patients who experienced the event of interest and the others was assessed using the Chi-squared test for categorical variables, the $t$ test for continuous variables with normal distribution, and Wilcoxon's rank-sum test for continuous variables with non-normal distribution. Normality was assessed using the Kolmogorov-Smirnov test.

Kaplan-Meier curves were calculated to describe the event-free survival of patients, both overall and by treatment groups. The log-rank test and Wilcoxon's test were used to assess the significance of differences in survival. $P<0.05$ was considered statistically significant.

All of the analyses were assessed using SAS v9.4 (SAS Institute Inc., Cary, NC, USA.).

\section{Compliance with ethical standards}

The authors assert that all of the procedures contributing to this work comply with the ethical standards of the relevant national and institutional committees on human experimentation and the Helsinki Declaration of 1975 as revised in 2008. This article does not contain any studies of human or animal subjects performed by any of the authors. Since this analysis was based on anonymous administrative data, patient informed consent and Ethical Committee approval were not required in Italy. As concerns the analysis that was conducted on electronic clinical chart records, patients admitted to our Hospital were asked to sign an informed consent for using their own data for research purpose. 


\section{Results}

\section{AAV epidemiology by integrated analysis of the administrative databases}

Between the years 2013 and 2018, 103 patients with diagnostic code for AAV were identified in FVG, accounting for 8.58 cases $/ 100000$ inhabitants (Table 1). Among these, patients with at least one hospitalization or death were 74/103 (71.8\%). 7/103 (6.8\%) died during the observation period. The whole number of hospitalizations was 285 in 74 patients (rate of 57 events per year), with an inpatient prevalence of 23.2 cases per 100000 admissions. Fiftyfive out of 74 (74.3\%) patients experienced more than one hospitalization. In most cases, the cause of hospitalization was a condition with high probability of being secondary to vasculitis $(119 / 285,41.8 \%)$ or the disease itself in more than half of cases $(62 / 119,52.1 \%$, ICD-9-CM code 446.4, "Wegener's granulomatosis") (Fig. 1). Infections were the second cause of hospitalization $(26 / 285,9.1 \%)$ (Fig. 1). In 10/103 patients (9.7\%), end-stage renal disease (ESRD) was recorded as an event. The presence of at least one positivity for ANCA antibodies was documented in
76/103 patients (73.8\%), mainly in patients carrying GPA. Globally, ANCA positivity tended to be associated with a greater likelihood of an event (hospitalization/death) (HR $1.75,95 \%$ CI $0.97-3.16, p=0.06$, Log-Rank test), irrespectively from disease diagnosis and after correction for age and gender. The first event occurred in 50\% of ANCApositive patients within 180 days (95\% CI 84-297) from the diagnosis, while in $50 \%$ of ANCA-negative patients in 859 days (95\% CI 87-1695). Notably, six out of the seven deaths occurred in ANCA-positive patients.

For the province of Udine, 530000 inhabitants served by the University Hospital of Udine and by the reference third-level center for rare rheumatologic diseases; laboratory results have been available since 2010 . Thus, we evaluated the cost of illness in patients suffering from AAV and residents in the province of Udine from 2010 to 2018. In the province, from 2010 to 2018, 57 patients (201 patientyears) with AAV were identified. GPA, EGPA, and MPA were diagnosed in 20 (35.1\%), 21 (36.8\%), and 16 (28.1\%) patients, respectively. They were ANCA-positive in 44/57 (77.2\%); of which GPA, EGPA, and MPA were diagnosed in 18 (40.9\%), 15 (34.1\%), and 11 (25\%) patients, respectively. The mean age at diagnosis was 54.5 (17.5) years. Again, the disease itself was the main cause of hospitalization in

Table 1 Comparison between Regional Health Information System of Friuli Venezia Giulia and clinical electronic chart records from our Hospital Clinic

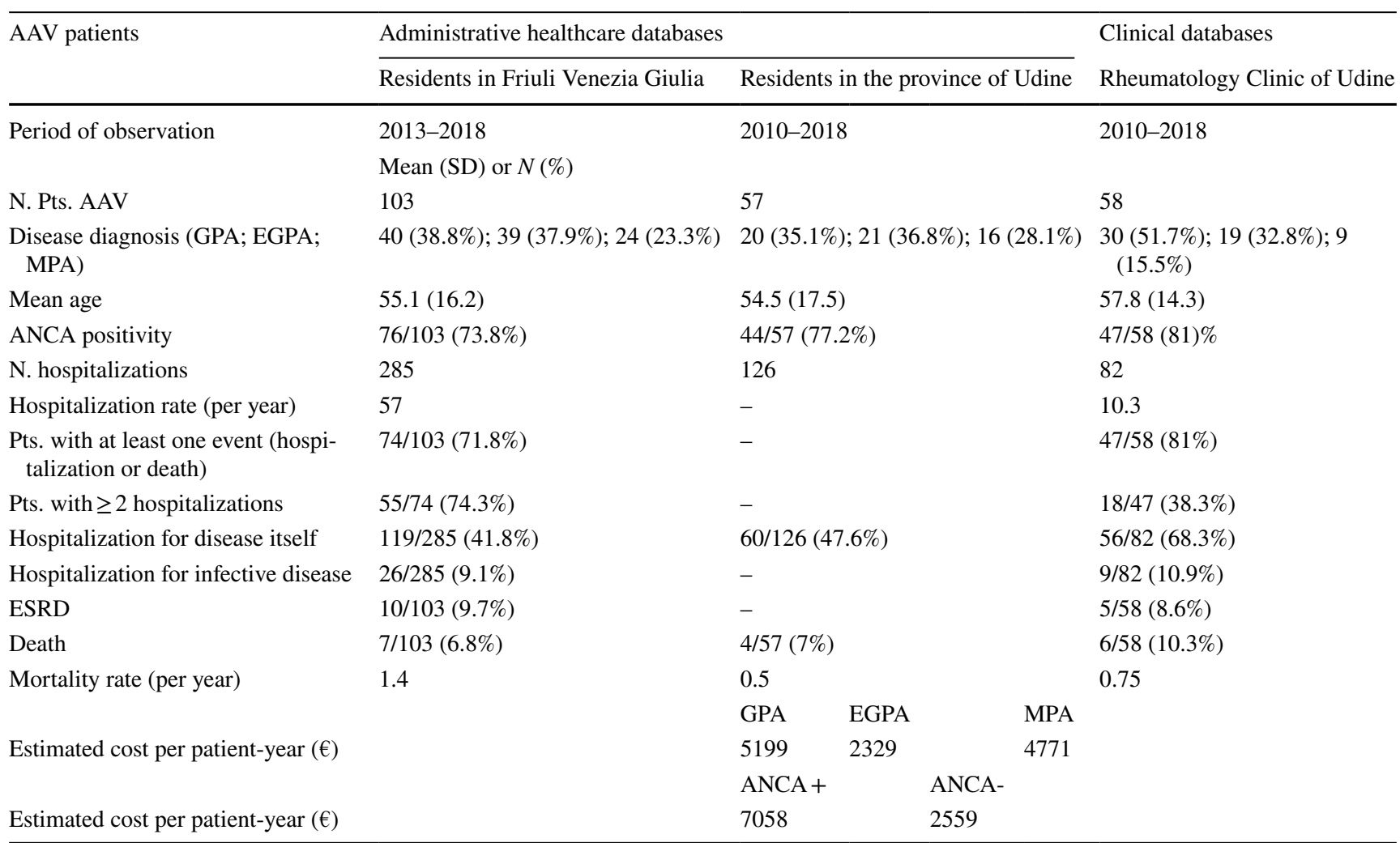

$A A V$ ANCA-associated vasculitis, $A N C A$ anti-neutrophil cytoplasmic antibodies, ESRD end-stage renal disease 


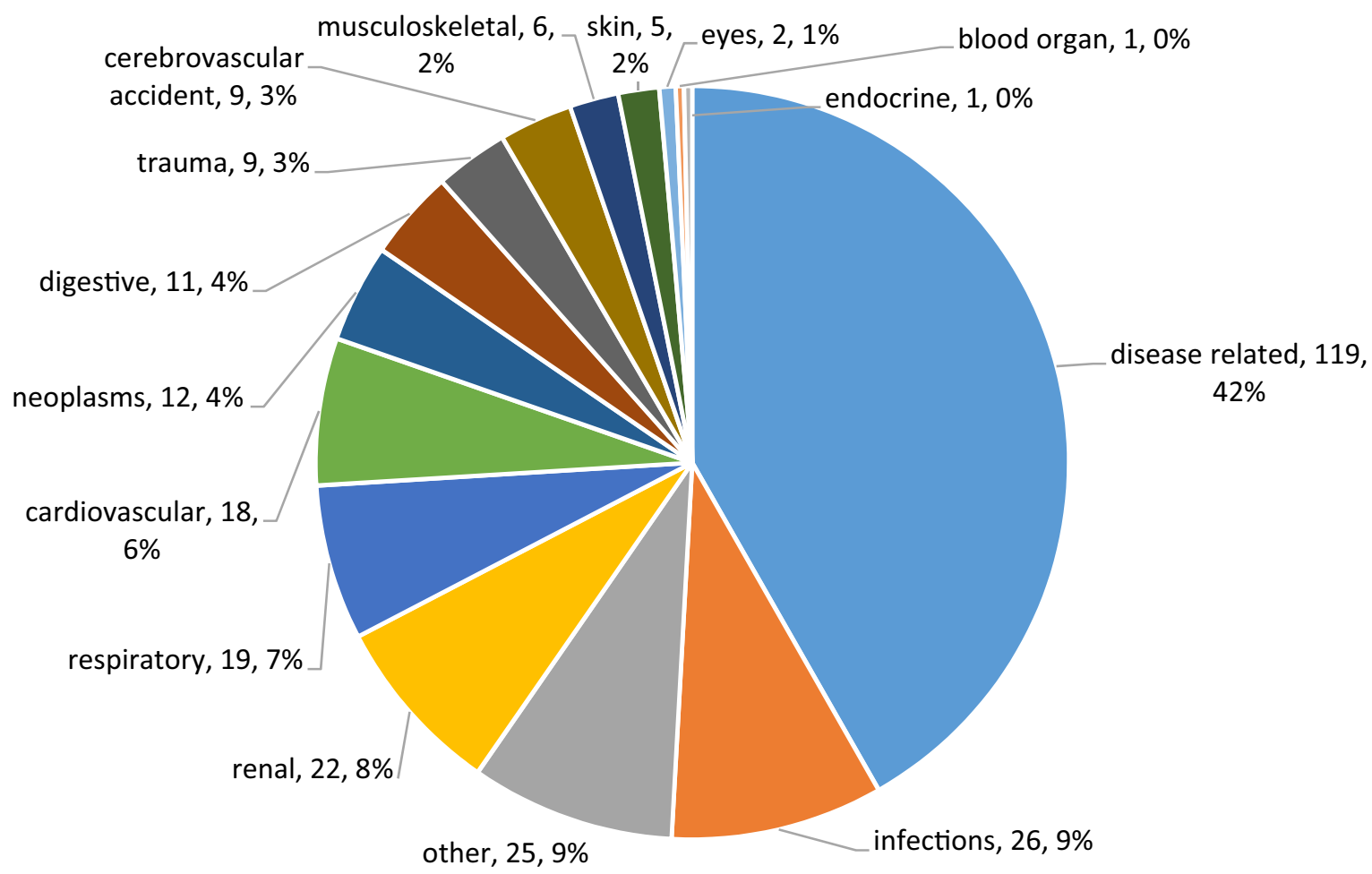

Fig. 1 Distribution of the categories of main discharge diagnosis for 285 hospitalizations of patients with AAV in FVG (years 2013-2018). the following ICD-9-CM codes were considered "disease related": 287.0 "Allergic purpura", 288.3 "Eosinophilia", 381.4 "Nonsuppurative otitis media, not specified as acute or chronic", 423.0 "Hemopericardium ", 437.4 "Cerebral arteritis", 446.4 "Wegener's granulomatosis", 447.6 "Arteritis, unspecified", 473.0 "Chronic maxillary sinusitis", 473.2 "Chronic ethmoidal sinusitis", 518.3 "Pulmonary eosinophilia", 580.4 "Acute glomerulonephritis with lesion of rapidly progressive glomerulonephritis", 582.4

almost half of the hospital discharges (60/126, 47.6\%). Four patients died during the observation period due to vasculitis itself (1/4), pneumonia (2/4), or haematological malignancy (1/4). Time to the first event (hospitalization or death) was significantly lower in ANCA-positive AAV patients than in ANCA-negative AAV patients, ANCA-positive AAV patients showing a three times higher risk than ANCAnegative patients (HR 3.38 95\% CI 1.13-10.18, $p=0.03$ ). Total estimated cost was $€ 1,215,078$, corresponding to $€ 6,168$ per patient-year. Costs for ANCA-positive AAV patients were much higher than for ANCA-negative patients $(€ 1,115,253$ vs $€ 99,825$, and $€ 7,058$ per person-year vs $€ 2,559$ per person-year, respectively) (Fig. 2). GPA and MPA showed the highest costs if compared to EGPA [GPA: $€ 239,168$ ( $€ 5,199$ per person-year) vs MPA: $€ 281,502$ ( $€$ 4,771 per person-year) vs EGPA: $€ 214,287$ (2,329 per person-year), respectively] (Fig. 3). Interestingly, hospital medications and medications dispensed by hospital pharmacies for EGPA patients represent a minor burden of expenditure as compared to MPA and GPA, whereas costs for prescribed
"Chronic nephritis with lesion of necrotizing glomerulitis", 582.9 "Chronic glomerulonephritis with unspecified pathological lesion in kidney", 584.5 "Renal failure with (acute) tubular necrosis", 375.53 "Stenosis of lacrimal canaliculi", 375.56 "Stenosis of nasolacrimal duct, acquired", 381.10 "Chronic serous otitis media, simple or unspecified", 420.90 "Acute pericarditis, unspecified", 446.29 "Other specified hypersensitivity angiitis", 478.74 "Stenosis of larynx", 519.19 "Unspecified disease of respiratory system", 582.89 "Chronic glomerulonephritis with lesion of: exudative nephritis"

medications are higher for EGPA patients (Fig. 3). Finally, costs for hospitalization were the highest among the direct costs of illness [ $€ 734,957$ ( $€ 3,731$ per person-year) vs other costs $€ 480,121$ ( $€ 2,437$ per person-year)].

\section{AAV epidemiology based on local clinical electronic chart records}

Further detailed information on patients with AAV was obtained using data from the Rheumatology Clinic of Udine, Referral Centre for AAV in FVG. Using our database, 58 patients (34 females, 24 males) with AAV diagnosis from 2010 to 2018 were identified. The mean age at the diagnosis of disease was 57.8 (14.3) years. Patients' diagnosis included GPA in 30 cases (51.7\%), EGPA in 19 cases $(32.8 \%)$, and MPA in 9 cases (15.5\%). They were ANCA-positive in $47 / 58$ (81\%), in particular 24/47 (51.1\%) cANCA/PR3 and 23/47 (48.9\%) pANCA/MPO. The main characteristics of the patients are summarized in Table 2. Patients with at least one hospitalization were $47 / 58$ (81\%). Eighteen out of 47 


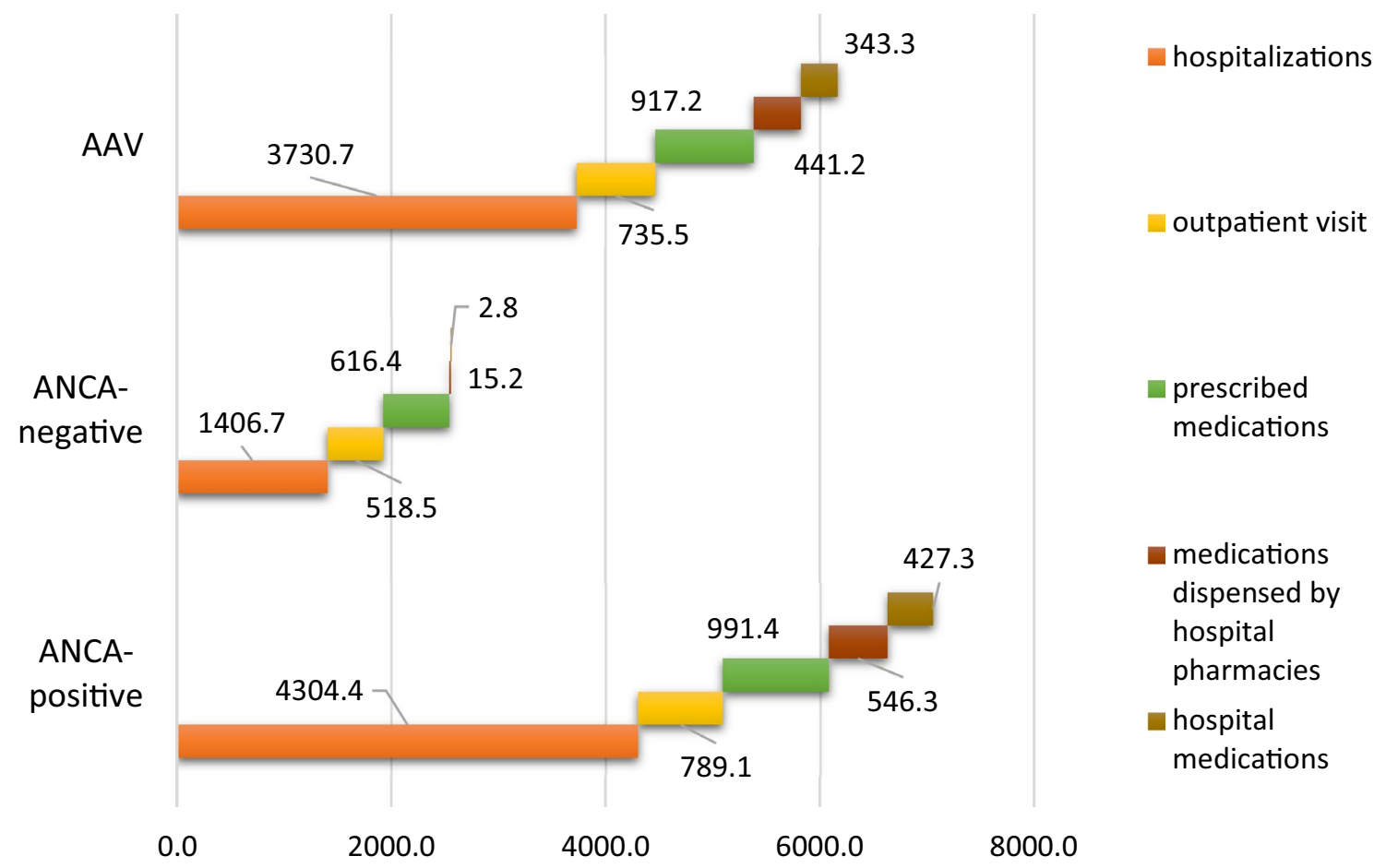

Fig. 2 The overall estimated healthcare costs $(€)$ per person-year for 57 AAV patients, residents in the province of Udine from 2010 to 2018 , divided into ANCA-positive and ANCA-negative

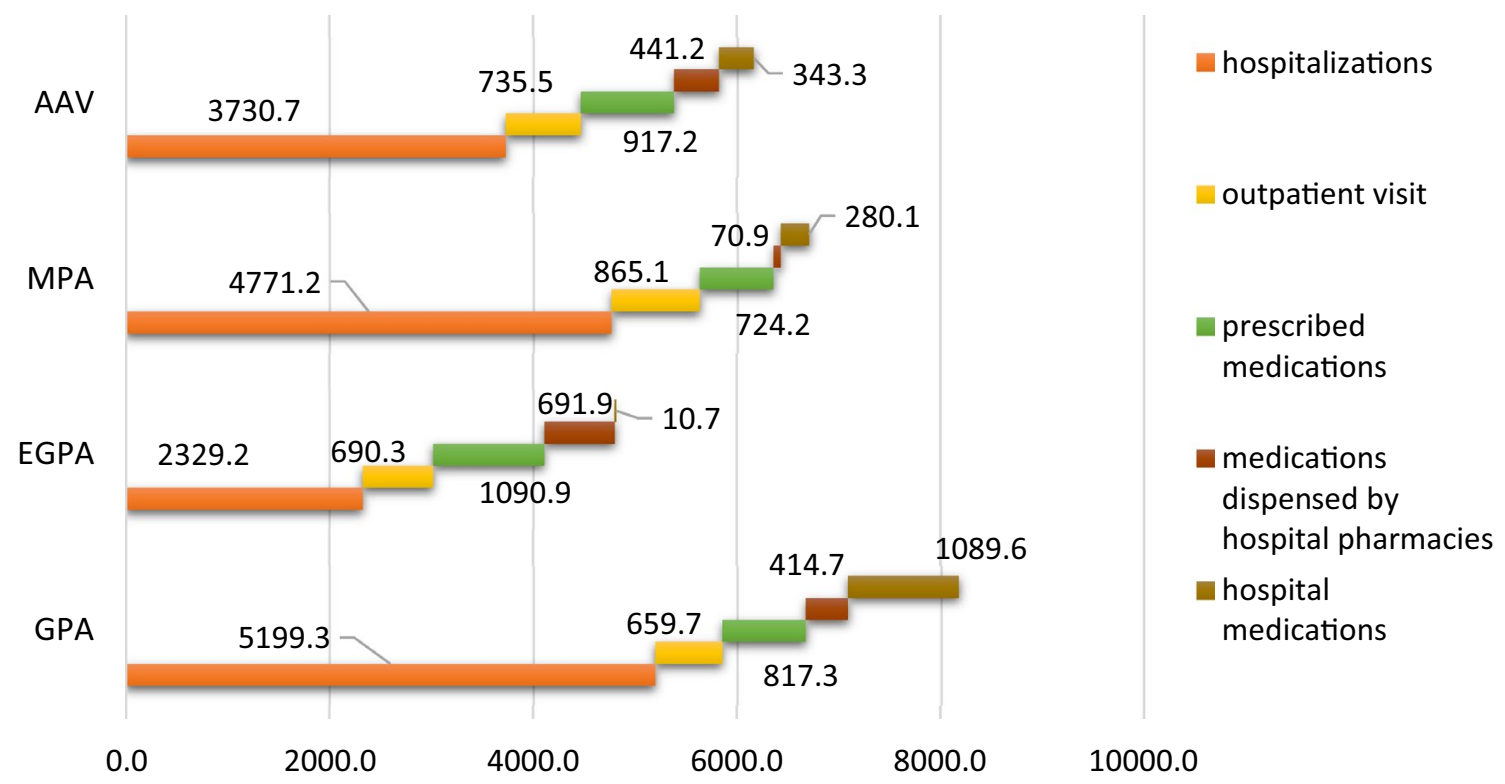

Fig. 3 The overall estimated healthcare costs $(€)$ per person-year for 57 AAV patients, residents in the province of Udine from 2010 to 2018 , also divided into each entity (GPA, MPA, and EGPA)

(38.3\%) patients experienced more than one hospitalization. The whole number of hospitalizations was 82 in 47 patients (10.3 events per year). The disease itself was the main cause of hospitalization in more than half of the hospital discharges [56/82 (68.3\%)]; infections were the second one
[9/82 (10.9\%)]. 5/58 (8.6\%) patients developed ESRD and $6 / 58(10.3 \%)$ patients died during the observation period. ANCA positivity seems to be associated with a greater likelihood of an event, i.e., hospitalization [67/82 (81.7\%)] and death [5/6 (83.3\%)]. Interestingly, patients becoming 
Table 2 Characteristics of 58 AAV patients followed by the Rheumatology Clinic of Udine (2010-2018)

\begin{tabular}{|c|c|}
\hline \multicolumn{2}{|l|}{ Demography } \\
\hline Age, years & $57.8 \pm 14.3$ \\
\hline Female gender & $34(58.6 \%)$ \\
\hline \multicolumn{2}{|l|}{ AAV diagnosis } \\
\hline GPA & $30(51.7 \%)$ \\
\hline EGPA & $19(32.8 \%)$ \\
\hline MPA & $9(15.5 \%)$ \\
\hline BVAS v3 & $13.9 \pm 6.7$ \\
\hline \multicolumn{2}{|c|}{ Baseline vasculitis involvement } \\
\hline Constitutional symptoms & $47(81 \%)$ \\
\hline ENT & $35(60.3 \%)$ \\
\hline Lungs & $29(50 \%)$ \\
\hline Kidney & $19(32.8 \%)$ \\
\hline Nervous system & $19(32.8 \%)$ \\
\hline Skin & $19(32.8 \%)$ \\
\hline Cardiovascular & $6(10.3 \%)$ \\
\hline Gastrointestinal & $6(10.3 \%)$ \\
\hline Eye & $2(3.4 \%)$ \\
\hline \multicolumn{2}{|l|}{ ANCA status } \\
\hline Positive ANCA & $47(81 \%)$ \\
\hline cANCA/PR3 & $24(51.1 \%)$ \\
\hline pANCA/MPO & $23(48.9 \%)$ \\
\hline
\end{tabular}

$A A V$ ANCA-associated vasculitis, GPA granulomatosis with polyangiitis, EGPA eosinophilic granulomatosis with polyangiitis, $M P A$ micropolyangiitis, BVAS Birmingham Vasculitis Activity Score, $E N T$ ear-nose-throat involvement, $A N C A$ anti-neutrophil cytoplasmic antibodies, $P R 3$ proteinase 3, MPO myeloperoxidase. Variables are reported as mean $\pm \mathrm{SD}$ or $N(\%)$

ANCA-negative showed a better outcome than persistently ANCA-positive patients did (Fig. 4).

\section{Discussion}

In the present study, we performed a global assessment of inpatient epidemiology and the economic burden of AAV in FVG (around 1200000 people) by integrating information coming from different sources, i.e., administrative healthcare databases and clinical databases (Table 1). This comparative analysis should be relevant for highlighting strengths and limitations of each source of data, and for addressing future researches in this field. Notably, cost estimation reported by us represents the first data available in the literature coming from a European healthcare system.

The management of AAV has evolved considerably in the recent decades, allowing improvements in outcomes and overall survival [19-22]. Nevertheless, the disease itself and, importantly, superimposed infections are still the main causes of hospitalization in AAV [3, 23]. In addition, still in the last decade, almost $10 \%$ of patients develop ESRD during the follow-up. Both the methodological approaches of our study led to this observation. Thus, the unmet needs of early diagnosis, in particular for renal involvement, and novel treatments, especially carrying significant steroidsparing effect, clearly emerged from these results [24, 25]. Second, the ANCA status was confirmed to be an important prognostic factor $[14,26]$. ANCA-positive patients, in particular ANCA/PR3-positive patients, more frequently show a relapsing-remitting disease [14]. Mortality and hospitalization rate were both significantly related to the presence of ANCA in our study. In addition, ANCA-positive patients showed a much shorter time to the first hospitalization than ANCA-negative patients, thus implying a more aggressive systemic disease and related higher level of immunosuppression. Again, both administrative data and clinical evaluation were strictly concordant on this point (Table 1). By contrast, the number of hospitalizations, even if in a different period, significantly differed between the administrative source and the clinical source of data, with the latter likely underestimating the events. This highlights the possible bias coming from clinical charts, especially when the time of observation is very long (i.e., 8 years), and, on the other hand, it reinforces the validity of an integrated approach by combining administrative databases and clinical evaluation in long-term retrospective observational studies on rare diseases. Also, the lower prevalence which we reported of MPA, which is often an isolated renal vasculitis managed by nephrologists, reinforces this concept.

The total cost of illness in patients suffering from AAV and residents in the province of Udine from 2010 to 2018 was $€ 1,215,078$, corresponding to $€ 6168$ patient-year. It was more than two times higher than that of patients with giant cell arteritis in the same area [27]. This estimation was the first one reported in the literature coming from a European healthcare system. The universal Italian healthcare system follows a tax-funded model similar to the Beveridge type, which is completely different from the insurance-based systems. With that premise, the substantially higher hospital costs recently reported by Ungprasert et al. from USA are not comparable with our results [3]. The main component of the total cost was represented by costs for hospitalization, amounting to $€ 734,957$ ( $€ 3731$ per person-year). Notably, higher costs of illness for ANCA-positive patients were documented for the first time (Fig. 2) as well as for GPA and MPA if compared to EGPA (Fig. 3) (GPA: $€ 5199$ per person-year vs MPA $€ 4771$ per person-year vs EGPA 2329 per person-year, respectively), therefore implying a higher number of patients with limited disease or with a more favourable course of vasculitis in EGPA than in GPA or MPA [1, 28-31]. However, the association between EGPA with chronic asthma in the adult, that is often resistant to many treatments, may explain the higher costs for prescribed 


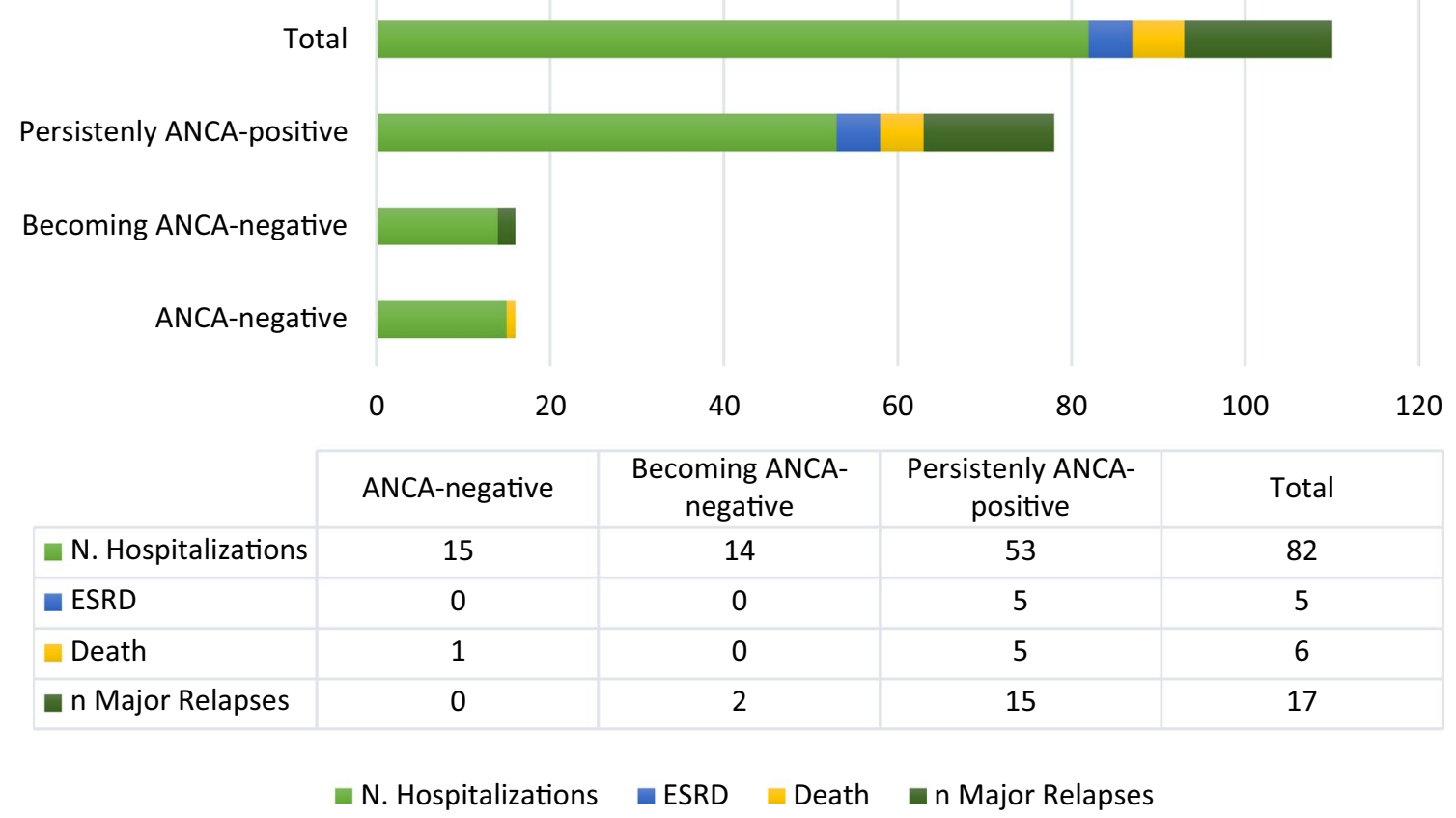

Fig. 4 Numbers of events in the 58 AAV patients followed by the Rheumatology Clinic of Udine (2010-2018). The patients have been divided into three categories: patients persistently ANCA-positive, patients becoming ANCA-negative, and patients ANCA-negative

medications for EGPA patients. After vasculitis resolution, asthma remains severe in up to $50 \%$ of patients and incidence of isolated-asthma and rhinosinus exacerbations remains constantly high. In addition, in EGPA patients with asthma, long-term severe or uncontrolled asthma is associated with baseline pulmonary and ear, nose, and throat manifestations but not with typical vasculitic features [32]. This observation further supports the consideration of EGPA as a separate entity among AAV.

This healthcare burden was expected in FVG considering the becoming into a chronic disease of $\mathrm{AAV}$, thanks to improvements in treatment. Emerging evidence in other studies $[10,33]$ is the negative effect on patients' physical and mental quality of life (QoL) of AAV, comparable to other chronic diseases. Current vasculitis disease assessment tools (BVAS v3 and VDI) are only weakly correlated with measures and variations of QoL [34, 35]. Interesting prospects might be offered by AVV-patient-reported outcomes (PRO) questionnaires. The AAV-PRO questionnaire could be useful to assess the patient's perspective on the burden of disease [36].

\section{Strengths and limitations}

The main strength of this examination is the achievement of largely homogeneous and overlapping results using health administrative databases, including, of note, even the costs estimated for hospital medications for each patient, and smaller clinical charts. Moreover, our prevalence estimation by healthcare administrative databases (8-9 cases per 100000 individuals) was close to that reported in the literature of around 2-13 cases per 100000 individuals [37-39], as well as the inpatient prevalence of 23.2 cases per 100000 admissions was in line to Li et al. [17]. The advantage of using regional informatics healthcare administrative databases is to obtain available information on patients' outcomes as well as healthcare burden and costs in a long period, possibly minimizing missing data. The latter are not available with smaller clinical databases, which risk being less informative for retrospective observational studies comprising long-term follow-up. Although not in the present study area, validation studies on rheumatic diseases have been published with good confidence in systemic rheumatic diseases [40, 41]. In addition, a similar study performed in US applied the same methods [42].

A limitation of using administrative health databases is the impossibility to correlate the disease assessment tools, patients' perception of QoL, and work ability status, with recorded events. However, a complementary clinical evaluation would serve to overcome this limit of a depersonalized disease description. Moreover, a preliminary analysis on work ability status in AAV patients from our cohort has been recently reported, demonstrating the importance of such evaluation as complementary to disease activity and damage in AAV [43]. Also, our work did not allow us to attribute a different impact on costs to different treatment regimens. Probably, more information than that available 
from retrospective cohorts based on administrative databases would be required to assess the impact of different treatment strategies employed on cost of illness. Controlled studies could more properly address this issue, by avoiding possible confounders. In addition, even if we have access to ED records, they do not include any cost information, as stated in the methods, and thus, our analysis did not consider the economic burden related to ED admission.

\section{Conclusion}

Even if rare, AAV are diseases showing a high level of economic source consumption for the healthcare system. The disease itself and the infectious complications during treatment still remain the main causes of hospitalization and related costs. The ANCA status seems to influence the outcome and the costs of illness greatly. EGPA seems to be a separate entity, also for its healthcare cost profile. Overall, the integration between healthcare administrative databases and clinical charts may draw the best portrait of these rare diseases, when considering a long time of observation.

Acknowledgements Open access funding provided by Universit Ã degli Studi di Udine within the CRUI-CARE Agreement.

Author contributions LQ conceived the study which was designed with the contribution of FV. Material preparation, data collection, and analysis were performed by LQ, FV, ET, and SDV. The first draft of the manuscript was written by LQ and ET, and all authors commented on previous versions of the manuscript. All authors read and approved the final manuscript.

Funding This research received no external funding.

Availability of data and material The data that support the findings of this study are available on request from the corresponding author, [LQ].

\section{Compliance with ethical standards}

Conflicts of interest The authors declare that they have no conflict of interest.

Statement of human and animal rights All procedures performed in studies involving human participants were in accordance with the ethical standards of the institutional research committee and with the 1975 Helsinki Declaration and its later amendments or comparable ethical standards. All the patients included in the clinical database gave consent to use their own data for research purposes. This article does not contain any studies involving animals performed by any of the authors. For using anonymized administrative databases, no ethics approval is required.

Informed consent Informed consent was obtained from all individual participants included in the study.

Open Access This article is licensed under a Creative Commons Attribution 4.0 International License, which permits use, sharing, adaptation, distribution and reproduction in any medium or format, as long as you give appropriate credit to the original author(s) and the source, provide a link to the Creative Commons licence, and indicate if changes were made. The images or other third party material in this article are included in the article's Creative Commons licence, unless indicated otherwise in a credit line to the material. If material is not included in the article's Creative Commons licence and your intended use is not permitted by statutory regulation or exceeds the permitted use, you will need to obtain permission directly from the copyright holder. To view a copy of this licence, visit http://creativecommons .org/licenses/by/4.0/.

\section{References}

1. Geetha D, Jefferson JA (2020) ANCA-associated vasculitis: core curriculum 2020. Am J Kidney Dis Off J Natl Kidney Found 75:124-137. https://doi.org/10.1053/j.ajkd.2019.04.031

2. Berti A, Dejaco C (2018) Update on the epidemiology, risk factors, and outcomes of systemic vasculitides. Best Pract Res Clin Rheumatol 32:271-294. https://doi.org/10.1016/j. berh.2018.09.001

3. Ungprasert P, Koster MJ, Cheungpasitporn W et al (2020) Inpatient epidemiology and economic burden of granulomatosis with polyangiitis: a 10-year study of the national inpatient sample. Rheumatol Oxf Engl. https://doi.org/10.1093/rheumatology/keaa0 69

4. Monti S, Bond M, Felicetti M et al (2019) One year in review 2019: vasculitis. Clin Exp Rheumatol 37(Suppl 117):3-19

5. Arman F, Barsoum M, Selamet U et al (2018) Antineutrophil cytoplasmic antibody-associated vasculitis, update on molecular pathogenesis, diagnosis, and treatment. Int J Nephrol Renov Dis 11:313-319. https://doi.org/10.2147/IJNRD.S162071

6. Jennette JC, Falk RJ, Bacon PA et al (2013) 2012 revised international chapel hill consensus conference nomenclature of vasculitides. Arthritis Rheum 65:1-11. https://doi.org/10.1002/ art.37715

7. Mahr A, Katsahian S, Varet H et al (2013) Revisiting the classification of clinical phenotypes of anti-neutrophil cytoplasmic antibody-associated vasculitis: a cluster analysis. Ann Rheum Dis 72:1003-1010. https://doi.org/10.1136/annrheumdis-2012-20175 0

8. Scherlinger M, Mertz P, Sagez F et al (2020) Worldwide trends in all-cause mortality of auto-immune systemic diseases between 2001 and 2014. Autoimmun Rev. https://doi.org/10.1016/j.autre v. 2020.102531

9. Greco A, Marinelli C, Fusconi M et al (2016) Clinic manifestations in granulomatosis with polyangiitis. Int J Immunopathol Pharmacol 29:151-159. https://doi.org/10.1177/0394632015 617063

10. Benarous L, Terrier B, Laborde-Casterot H et al (2017) Employment, work disability and quality of life in patients with ANCAassociated vasculitides. The EXPOVAS study. Clin Exp Rheumatol 35(Suppl 103):40-46

11. Hassan RI, Gaffo AL (2017) Rituximab in ANCA-associated vasculitis. Curr Rheumatol Rep 19:6. https://doi.org/10.1007/s1192 6-017-0632-1

12. Raffray L, Guillevin L (2018) Treatment of eosinophilic granulomatosis with polyangiitis: a review. Drugs 78:809-821. https:// doi.org/10.1007/s40265-018-0920-8

13. Comarmond C, Cacoub P (2014) Granulomatosis with polyangiitis (Wegener): clinical aspects and treatment. Autoimmun Rev 13:1121-1125. https://doi.org/10.1016/j.autrev.2014.08.017 
14. McClure ME, Wason J, Gopaluni S et al (2019) Evaluation of PR3-ANCA status after rituximab for ANCA-associated vasculitis. J Clin Rheumatol Pract Rep Rheum Musculoskelet Dis 25:217-223. https://doi.org/10.1097/RHU.0000000000001030

15. Tieu J, Smith R, Basu N et al (2020) Rituximab for maintenance of remission in ANCA-associated vasculitis: expert consensus guidelines. Rheumatol Oxf Engl 59:e24-e32. https://doi.org/10.1093/ rheumatology/kez640

16. Guillevin L, Pagnoux C, Seror R et al (2011) The Five-Factor Score revisited: assessment of prognoses of systemic necrotizing vasculitides based on the French Vasculitis Study Group (FVSG) cohort. Medicine (Baltimore) 90:19-27. https://doi.org/10.1097/ MD.0b013e318205a4c6

17. Li J, Cui Z, Long J-Y et al (2018) The frequency of ANCA-associated vasculitis in a national database of hospitalized patients in China. Arthritis Res Ther 20:226. https://doi.org/10.1186/s1307 5-018-1708-7

18. Ministero della Salute. Elenco malattie rare esentate dalla partecipazione al costo. Allegato 7 DPCM 12 gennaio 2017. https://www.trovanorme.salute.gov.it/norme/ renderPdf.spring?seriegu $=S G \&$ datagu $=18 / 03 / 2017 \&$ redaz $=17 \mathrm{~A} 02015 \&$ art $\mathrm{p}=12 \&$ art $=1 \& \mathrm{sub}$ ar $\mathrm{t}=1 \& \mathrm{sub}$ ar $\mathrm{t} 1=10 \&$ vers $=1 \&$ prog $=001$. Accessed 24 April 2018

19. Wallace ZS, Miloslavsky EM (2020) Management of ANCA associated vasculitis. BMJ 368:m421. https://doi.org/10.1136/ bmj.m421

20. Heijl C, Mohammad AJ, Westman K, Höglund P (2017) Longterm patient survival in a Swedish population-based cohort of patients with ANCA-associated vasculitis. RMD Open 3:e000435. https://doi.org/10.1136/rmdopen-2017-000435

21. McClure M, Gopaluni S, Jayne D, Jones R (2018) B cell therapy in ANCA-associated vasculitis: current and emerging treatment options. Nat Rev Rheumatol 14:580-591. https://doi.org/10.1038/ s41584-018-0065-x

22. Emejuaiwe N (2019) Treatment strategies in ANCA-associated vasculitis. Curr Rheumatol Rep 21:33. https://doi.org/10.1007/ s11926-019-0835-8

23. Wallace ZS, Lu N, Miloslavsky E et al (2017) Nationwide trends in hospitalizations and in-hospital mortality of granulomatosis with polyangiitis. Arthritis Care Res 69:915-921. https://doi. org/10.1002/acr.22976

24. Walsh M, Merkel PA, Peh C-A et al (2020) Plasma exchange and glucocorticoids in severe ANCA-associated vasculitis. N Engl J Med 382:622-631. https://doi.org/10.1056/NEJMoa1803537

25. Bajema IM, Bruijn JA, Casian A et al (2017) The European vasculitis society 2016 meeting report. Kidney Int Rep 2:1018-1031. https://doi.org/10.1016/j.ekir.2017.09.008

26. Bulanov NM, Makarov EA, Shchegoleva EM et al (2018) Relationship between serologic profile (ANCA type) and clinical features of renal involvement in ANCA-associated vasculitides. Ter Arkh 90:15-21. https://doi.org/10.26442/terarkh201890615-21

27. Valent F, Bond M, Cavallaro E et al (2020) Data linkage analysis of giant cell arteritis in Italy: Healthcare burden and cost of illness in the Italian region of Friuli Venezia Giulia (2001-2017). Vasc Med. 25:150-156. https://doi.org/10.1177/1358863X19886074

28. Geetha D, Jin Q, Scott J et al (2018) Comparisons of guidelines and recommendations on managing antineutrophil cytoplasmic antibody-associated vasculitis. Kidney Int Rep 3:1039-1049. https ://doi.org/10.1016/j.ekir.2018.05.007

29. Mahr A, Moosig F, Neumann T et al (2014) Eosinophilic granulomatosis with polyangiitis (Churg-Strauss): evolutions in classification, etiopathogenesis, assessment and management. Curr Opin Rheumatol 26:16-23. https://doi.org/10.1097/BOR.00000 00000000015
30. McKinney EF, Willcocks LC, Broecker V, Smith KGC (2014) The immunopathology of ANCA-associated vasculitis. Semin Immunopathol 36:461-478. https://doi.org/10.1007/s00281-014-0436-6

31. Cornec D, Gall EC-L, Fervenza FC, Specks U (2016) ANCAassociated vasculitis - clinical utility of using ANCA specificity to classify patients. Nat Rev Rheumatol 12:570-579. https://doi. org/10.1038/nrrheum.2016.123

32. Berti A, Cornec D, Casal Moura M, Smyth RJ, Dagna L, Specks U, Keogh KA (2020) Eosinophilic granulomatosis with polyangiitis: clinical predictors of long-term asthma severity. Chest 157(5):1086-1099. https://doi.org/10.1016/j.chest.2019.11.045

33. Hinojosa-Azaola A, Jiménez-González A, Alcocer-Castillejos N (2018) Patient and physician perspectives on the impact of healthrelated quality of life in Mexican patients with ANCA-associated vasculitis. Rheumatol Int 38:631-640. https://doi.org/10.1007/ s00296-017-3904-1

34. Exley AR, Bacon PA, Luqmani RA et al (1997) Development and initial validation of the Vasculitis damage index for the standardized clinical assessment of damage in the systemic vasculitides. Arthritis Rheum 40:371-380. https://doi.org/10.1002/art.17804 00222

35. Mukhtyar C, Lee R, Brown D et al (2009) Modification and validation of the birmingham vasculitis activity score (version 3). Ann Rheum Dis 68:1827-1832. https://doi.org/10.1136/ ard.2008.101279

36. Robson JC, Dawson J, Doll H et al (2018) Validation of the ANCA-associated vasculitis patient-reported outcomes (AAVPRO) questionnaire. Ann Rheum Dis 77:1157-1164. https://doi. org/10.1136/annrheumdis-2017-21271

37. Watts RA, Mahr A, Mohammad AJ et al (2015) Classification, epidemiology and clinical subgrouping of antineutrophil cytoplasmic antibody (ANCA)-associated vasculitis. Nephrol Dial Transplant 30:i14-i22

38. Pierini FA, Scolnik M, Scaglioni V, Mollerach F, Soriano ER (2019) Incidence and prevalence of granulomatosis with polyangiitis and microscopic polyangiitis in health management organization in Argentina: a 15-year study. Clin Rheumatol 38:1935-1940

39. Watts RA, Al-Taiar A, Scott DG, Macgregor AJ (2009) Prevalence and incidence of Wegener's granulomatosis in the UK General Practice Research Database. Arthritis Rheum 61:1412-1416

40. Bernatsky S, Linehan T, Hanly JG (2011) The accuracy of administrative data diagnoses of systemic autoimmune rheumatic diseases. J Rheumatol 38(8):1612-1616. https://doi.org/10.3899/ jrheum.101149

41. Bernatsky S, Panopalis P, Pineau CA, Hudson M, St Pierre Y, Clarke AE (2011) Healthcare costs of inflammatory myopathies. J Rheumatol 38(5):885-888. https://doi.org/10.3899/jrheum.10108 3

42. Raimundo K, Farr AM, Kim G, Duna G (2015) Clinical and economic burden of antineutrophil cytoplasmic antibody-associated vasculitis in the United States. J Rheumatol 42(12):2383-2391. https://doi.org/10.3899/jrheum.150479

43. Bond M, Quartuccio L, Zandonella S, De Vita S (2019) 234 ANCA-associated vasculitis and work ability: evaluation of disease impact on working life as an outcome measure in a monocentric Italian cohort. Rheumatology (Oxford) 58(2):104. https:// doi.org/10.1093/rheumatology/kez062.008

Publisher's Note Springer Nature remains neutral with regard to jurisdictional claims in published maps and institutional affiliations. 